\title{
Estructura y estado de conservación de los bosques de Prosopis flexuosa D.C. (Fabaceae, subfamilia: Mimosoideae) en el noreste de Mendoza (Argentina)
}

\author{
Structure and conservative condition of the Prosopis flexuosa D.C. (Fabaceae, subfamily: \\ Mimosoideae) woodlands in northeast Mendoza (Argentina)
}

\begin{abstract}
JUAN AGUSTÍN ALVAREZ ${ }^{1 *}$, PABLO EUGENIO VILLAGRA ${ }^{1}$, MARIANO ANÍBAL CONY², ERICA MARISA CESCA ${ }^{1} \&$ JOSÉ ARMANDO BONINSEGNA $^{1}$
\end{abstract}

\author{
${ }^{1}$ Departamento de Dendrocronología e Historia Ambiental, Instituto Argentino de Nivología, Glaciología y Ciencias \\ Ambientales (IANIGLA), Consejo Nacional de Investigaciones Científicas y Técnicas (CONICET), Casilla de Correo 330 , \\ 5500, Mendoza, Argentina \\ ${ }^{2}$ Instituto Argentino de Investigaciones de las Zonas Áridas (IADIZA), CONICET, Mendoza, Argentina; \\ * e-mail para correspondencia: jalvarez@lab.cricyt.edu.ar
}

\begin{abstract}
RESUMEN
La recomendación de normas de manejo que regulen el uso de los recursos forestales debe estar basada en el conocimiento de la estructura y dinámica de los mismos. El objetivo de este trabajo fue conocer la estructura poblacional de las principales unidades boscosas del bosque de Prosopis flexuosa del noreste de Mendoza, sus condiciones sanitarias y su potencial productivo. Se muestrearon un total de 1.471 algarrobos en las cuatro unidades boscosas más representativas. La densidad total de algarrobos fue la siguiente: bosque semicerrado de P. flexuosa con Atriplex lampa y Lycium tenuispinosum en valles intermédanos (Bosque 1): 181 árboles ha ${ }^{-1}$, bosque abierto de P. flexuosa con Trichomaria usillo y Suaeda divaricata en ondulaciones (Bosque 2): 155 árboles ha ${ }^{-1}$, bosque abierto de $P$. flexuosa con T. usillo (Bosque 3): 233 árboles ha ${ }^{-1}$ y bosque abierto de $P$. flexuosa con A. lampa en ondulaciones suaves (Bosque 4): 215 árboles ha ${ }^{-1}$. El análisis de componentes principales de la estructura diamétrica agrupó los sitios relevados en las distintas unidades boscosas según la proporción de árboles de diámetro basal mayor a $25 \mathrm{~cm}$. Los sitios del Bosque 1 (mayor proporción de árboles grandes), se separaron de los sitios con mayor proporción árboles pequeños (bosques 2 y 4). Debido al hábito de crecimiento de los algarrobos, la cantidad de productos maderables de estos bosques es baja. Además, $P$. flexuosa presenta en el área un porcentaje alto de individuos con más de dos fustes, la forma en muchos casos es decumbente y la altura de los fustes es menor a un metro. Por lo tanto, el potencial forestal del bosque es bajo y el posible aprovechamiento debería realizarse a escala local, considerando la inclusión de otras actividades complementarias en zonas establecidas para tal fin.
\end{abstract}

Palabras clave: desierto del Monte, estructura del bosque, Prosopis flexuosa, Reserva Telteca, productos maderables.

\section{ABSTRACT}

The recommendation of management rules that regulate the use of forest resources must be based on the knowledge of the structure and dynamics of forests. This work allowed to find out about the population structure of the Prosopis flexuosa woodlands of the northeast of Mendoza, as well as their sanitary conditions and the management potential. A total of 1,471 Prosopis individuals were sampled in the four most representative woodlands units. The total Prosopis density was as follows: P. flexuosa semi-closed woodland with Atriplex lampa and Lycium tenuispinosum in valleys in-between dunes (Woodland 1): 181 trees ha ${ }^{-1} ; P$. flexuosa open woodland with Trichomaria usillo and Suaeda divaricata in the undulations (Woodland 2): 155 trees ha ${ }^{-1} ;$ P. flexuosa open woodland with T. usillo (Woodland 3): 233 trees ha ${ }^{-1}$, and P. flexuosa open woodland with A. lampa in soft undulations (Woodland 4): 215 trees ha ${ }^{-1}$. The principal components analysis on the diametric structure grouped the sites surveyed in the different woodland units as per the proportion of individuals with larger basal diameter. The sites from Woodland 1 (larger proportion of big trees), were separated from the sites with a higher proportion of small trees (Woodland 2 and 4). Due to the Prosopis growth habit, the quantity of wood products from these forests is low. Besides P. flexuosa presents in the area a high percentage of individuals with more than two stems, the tree shape is generally decumbent and the bole height is less than a meter. Therefore, the possible use should be done at local scale, taking into account the inclusion of other complementary activities.

Key words: Monte desert, woodland structure, Prosopis flexuosa, Telteca Reserve, wood products. 


\section{INTRODUCCIÓN}

La recomendación de normas de manejo que regulen el uso de los recursos forestales debe estar basada en el conocimiento de la estructura y dinámica de los mismos. Los bosques de zonas áridas han sido poco estudiados desde el punto de vista de sus posibilidades de manejo sustentable, por lo que generalmente han sido utilizados con un criterio extractivo, sin tener en cuenta la tasa de regeneración de los recursos. Sin embargo, es posible el manejo de la vegetación nativa en regiones áridas a través de la utilización de las poblaciones existentes, altamente adaptadas a las características extremas del ambiente, atendiendo a su preservación y al incremento de su productividad. El uso sin conocimiento de dichas especies provoca efectos ambientales negativos en regiones donde las relaciones ecológicas son frágiles y sensibles a los cambios. Los bosques de zonas áridas tienen un bajo potencial de producción de madera, pero suministran a las poblaciones locales una variada gama de productos no madereros y servicios ambientales (FAO 1999). Argentina posee más del $60 \%$ de su territorio en ambientes áridos o semiáridos, de los cuales 46 millones de hectáreas corresponden a la Provincia Fitogeográfica del Monte.

El algarrobo dulce, Prosopis flexuosa D.C. (Fabaceae, Mimosoideae), es la principal especie arbórea del Monte, funcionando como estructuradora de las comunidades del desierto (Roig 1993, Rossi \& Villagra 2003). Los bosques abiertos de $P$. flexuosa que se ubican en las llanuras orientales mendocinas soportan extremas condiciones ambientales, entre $100 \mathrm{y}$ $350 \mathrm{~mm}$ de precipitación media anual (Telteca al noreste y General Alvear al sureste respectivamente), con déficit hídrico durante la mayor parte del año. Los bosques de algarrobo de Mendoza, son desde hace varios siglos fuente de recursos y materiales para el desarrollo de los distintos modelos agrícolas de las áreas irrigadas y usos diversos dentro de la ciudad de Mendoza y poblaciones aledañas. A fines del siglo XIX, la llegada del ferrocarril y la sustitución del modelo económico desencadenaron la tala intensiva del bosque nativo. Esta práctica provocó en la zona de estudio la desestructuración del ambiente y el comienzo de procesos de desertificación (Abraham \& Prieto
1999). Actualmente, se encuentra prohibida la extracción forestal de cualquier tipo (Gobierno de Mendoza 1997a, 1997b, 1997c, 1997d), medida que se tomó con un criterio de precaución con el objeto de evitar que se siga degradando el bosque, aunque sin una evaluación técnica adecuada sobre las posibilidades productivas de la zona. Esta prohibición de uso de leña es razonable teniendo en cuenta el estado de degradación del bosque y la falta de conocimiento de su dinamismo. Sin embargo, la posibilidad de uso dependería directamente del avance en el conocimiento de su estructura y dinámica, teniendo en cuenta la calidad de vida de los pobladores que habitan en estos bosques abiertos.

El conocimiento de los parámetros poblacionales de los bosques del noreste de Mendoza permitirá evaluar el estado actual de los mismos, la disponibilidad de productos forestales y contar con una base para la comprensión de la capacidad de recuperación del sistema, su evolución histórica y la estructura futura de los mismos. Los objetivos de este trabajo fueron estudiar la estructura actual y estado de conservación de las comunidades boscosas del noreste de Mendoza, y contribuir a la determinación de la potencialidad de manejo de estos bosques.

\section{MATERIALES Y MÉTODOS}

\section{Área de estudio}

Los bosques de algarrobo de la provincia de Mendoza se ubican en las llanuras al pie de la cordillera de los Andes. Estas planicies o "travesías", están divididas por los ríos Tunuyán y Diamante, formando tres llanuras distintas: al noreste la Travesía de Guanacache, en el centro la Travesía del Tunuyán y al sur la Travesía de la Varita (Roig et al. 1992). El área de estudio se encuentra en la Travesía de Guanacache, que se extiende desde los 600 a los $400 \mathrm{~m}$ de altitud y está formada por el aporte sedimentario de los ríos que traen materiales finos desde las montañas (Abraham 2000) (Fig. 1). El modelado eólico determina la existencia de médanos de hasta $20 \mathrm{~m}$ de alto (Altos Limpios) desprovistos totalmente de vegetación (González-Loyarte 1992). El área es climáticamente árida, con grandes amplitudes térmicas diarias y anuales, 


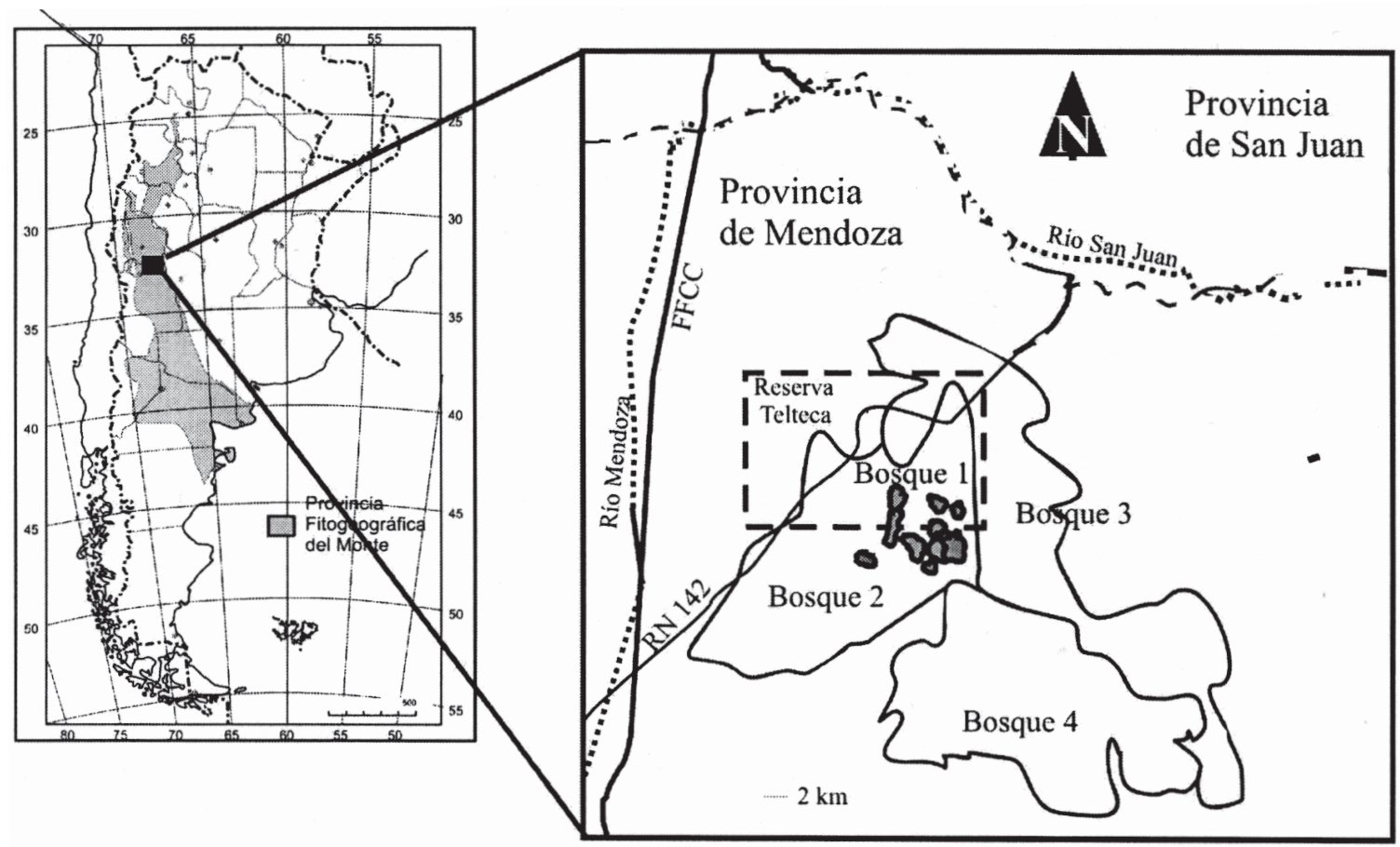

Fig. 1: Ubicación geográfica del área de estudio y ubicación de las principales unidades boscosas adaptado de González-Loyarte et al. (2000). RN 142: Ruta Nacional 142 y FFCC: Ramal Ferrocarril BAP.

Geographical location of the area of study and the principal woodland units, adapted from Gonzalez-Loyarte et al. (2000). RN 142: National Route 142 and FFCC: Branch Railroad BAP.

las precipitaciones se concentran en verano. La temperatura media anual es de $18,5{ }^{\circ} \mathrm{C}$, la temperatura máxima media es de $27{ }^{\circ} \mathrm{C}$ y la temperatura mínima media es de $9,3{ }^{\circ} \mathrm{C}$ (Estrella et al. 1979). Los registros anuales de precipitación en las dos estaciones meteorológicas más cercanas, indican 164,6 mm (Encón, San Juan) y 155,0 mm (El Retamo, Mendoza) (Estrella et al. 1979) (Fig. 2). Los vientos predominantes son del sureste. La profundidad de la napa freática varía entre 5 y $15 \mathrm{~m}$ (Roig et al. 1992). Fitogeográficamente esta región se incluye en el Monte, donde la vegetación está representada por la estepa de arbustos, comunidad dominada por zigofiláceas del género Larrea y Bulnesia retama, la estepa edáfica de halófitas y los bosques abiertos de Prosopis flexuosa en zonas con un suplemento de agua (Morello 1958, Villagra et al. 2004).

\section{Elección de las unidades de vegetación}

Se tomó como base para este trabajo el mapa de vegetación del noreste de Mendoza elaborado por González-Loyarte et al. (2000) a partir de una clasificación no supervisada de las bandas 3 , 4 y 5 de la imagen Landsat Thematic Mapper del 23 febrero de 1989 y corroboradas en el campo, a través del método fitosociológico (BraunBlanquet 1979). González-Loyarte et al. (2000) describen las unidades de vegetación de acuerdo a su composición florística y la abundancia relativa de las distintas especies. Entre las unidades definidas por estos autores se seleccionaron para este trabajo las cuatro unidades boscosas más representativas del área (Fig. 1): (1) bosque semicerrado de P. flexuosa con Atriplex lampa y Lycium tenuispinosum en valles intermédanos (Bosque 1). Se extiende desde los $67^{\circ} 54^{\prime} \mathrm{O}$ hacia el oeste y está limitado en su costado noroeste por la ruta nacional 142 , llega hasta el sur a los $32^{\circ} 28^{\prime} \mathrm{S}$; (2) bosque abierto de $P$. flexuosa con Trichomaria usillo y Suaeda divaricata en ondulaciones (Bosque 2). Ocupa las zonas medanosas que rodean los valles en los que se encuentra el bosque anterior; (3) el bosque abierto de P. flexuosa con T. usillo (Bosque 3), ocupa una franja de 2 a $12 \mathrm{~km}$ de ancho que va desde los $32^{\circ} 15^{\prime} \mathrm{S}$ en el norte hasta los $32^{\circ} 32^{\prime}$ S en la ubicación más 
meridional. Es una zona de médanos bajos que forman ondulaciones; (4) bosque abierto de $P$. flexuosa con Atriplex lampa en ondulaciones suaves (Bosque 4). Se distribuye principalmente hacia el sur de los bosques 2 y 3 .

\section{Dasonomía y estado de conservación}

En cada tipo de bosque se eligieron al azar los sitios de muestreo (unidades muestrales), donde se ubicaron tres parcelas de $1.000 \mathrm{~m}^{2}(50 \times 20$ m) separadas entre sí por una distancia de 100 m. Se muestrearon 30 sitios, nueve en el
Bosque 1, cuatro en el Bosque 2, nueve en el Bosque 3 y ocho en el Bosque 4.

En cada parcela se determinó el número de individuos adultos y juveniles. Se consideraron juveniles a los individuos cuyo diámetro basal fue menor a los $7,5 \mathrm{~cm}$. A su vez en cada árbol se registró: diámetro basal de cada fuste, altura del árbol, forma, dimensiones de la copa (diámetro mayor y menor), número de los fustes, altura de los fustes y estado sanitario. Se calculó el diámetro basal equivalente para cada árbol (que en adelante se denomina DAB en este trabajo), a través de la siguiente fórmula:



Fig. 2: Diagrama ombrotérmico según Bagnouls \& Gaussen (1957) para las estaciones meteorológicas de El Retamo $\left(67^{\circ} 28^{\prime} \mathrm{O}, 32^{\circ} 35^{\prime} \mathrm{S}\right)$ y Encón $\left(67^{\circ} 47^{\prime} \mathrm{O}, 32^{\circ} 15^{\prime} \mathrm{S}\right)$. Las dos muestran períodos de déficit hídrico (áreas rayadas) durante todo el año; $\mathrm{t}=$ temperatura, $\mathrm{p}=$ precipitación.

Ombrothermic diagram according to Bagnouls \& Gaussen (1957) for the meteorological stations of El Retamo $\left(67^{\circ} 28^{\prime}\right.$ W, $\left.32^{\circ} 35^{\prime} \mathrm{S}\right)$ y Encón $\left(67^{\circ} 47^{\prime} \mathrm{W}, 32^{\circ} 15^{\prime} \mathrm{S}\right)$. Both show periods of water deficit (stripped areas) during the whole year; $t=$ temperature, $\mathrm{p}=$ precipitation. 
$\mathrm{DAB}=2 \sqrt{\left\{\pi\left(\mathrm{dab}_{1} / 2\right)^{2}+\pi\left(\mathrm{dab}_{2} / 2\right)^{2}+\pi\left(\mathrm{dab}_{3} / 2\right)^{2}+\ldots .+\pi\left(\mathrm{dab}_{\mathrm{n}} / 2\right)^{2}\right\} / \pi}$

donde el $\mathrm{dab}_{1}, \mathrm{dab}_{2}, \ldots, \mathrm{dab}_{\mathrm{n}}$ es el diámetro basal de cada fuste. Se realizó un análisis cualitativo de la sanidad de los árboles encontrados, a los que se clasificó en: sanos, de sanidad regular, sanidad mala y muertos. Se incluyeron en la categoría "sano" a aquellos individuos en que todas las ramas estaban vivas y con buen vigor. Los de sanidad regular fueron aquellos árboles que presentaban menos del 50 $\%$ de ramas secas. Los individuos con más del $50 \%$ de ramas o fustes afectados, pero que poseen algunas ramas o fustes en buen estado sanitario se clasificaron como de sanidad mala.

En cuanto a la forma los algarrobos se clasificaron en: erectos, semierectos y decumbentes. Se consideraron erectos a los árboles con hábito de crecimiento vertical, semierectos a aquellos ejemplares multifustales o con fuste principal ramificado y cuyas ramas secundarias presentaran un crecimiento cercano a la horizontal o hacia abajo pero que no llegaran al suelo. Se consideraron decumbentes a los individuos multifustales y cuyas ramas laterales fueran péndulas de forma tal que alcanzaran el suelo (Pasiecznik et al. 2001). La forma y el estado sanitario de las unidades boscosas se analizaron para los individuos adultos (diámetro basal $>7,5 \mathrm{~cm}$ ).

\section{Clasificación de los productos maderables}

Con el objetivo de analizar el potencial de uso que tienen los bosques de la zona, en cada árbol se estimó visualmente la cantidad de productos maderables factibles de obtener. La clasificación de los mismos se llevó adelante con el criterio de utilización usado en la zona: vigas o madera para aserrío (trozos mayores 35 $\mathrm{cm}$ de diámetro superiores a los 1,2 $\mathrm{m}$ de largo), rodrigón (entre 25 y $35 \mathrm{~cm}$ de diámetro y $2,5 \mathrm{~m}$ de largo), postes (entre 20 y $25 \mathrm{~cm}$ de diámetro y 2,5 m de largo), $1 / 2$ poste (entre 20 y $25 \mathrm{~cm}$ de diámetro y hasta $2 \mathrm{~m}$ de largo), parralero (hasta $20 \mathrm{~cm}$ diámetro y 2,5 m de largo). Para su cuantificación se consideraron solo aquellos troncos o partes de los mismos que, sin ramificaciones, alcanzaran estas medidas. Se estimó la cantidad total de cada uno de estos productos por hectárea. La cantidad de leña presente en cada sitio no fue cuantificada en este estudio ya que no se disponen para la zona de curvas alométricas que permitan estimar el volumen total de madera de cada árbol. Se calculó el área basal total (a nivel del suelo), de los troncos de $P$. flexuosa en cada uno de los sitios muestreados, para relacionar este parámetro con el total de productos forestales registrados.

\section{Análisis de los datos}

Se utilizó estadística descriptiva para el análisis de los datos. Además, para comprobar si la clasificación previa obtenida del mapa de comunidades vegetales (González-Loyarte et al. 2000) está relacionada con la estructura poblacional del bosque, se realizó un análisis de componentes principales con el objeto de encontrar agrupamiento de los sitios en función de la estructura diamétrica. Si la clasificación previa se relacionara con la estructura poblacional entonces los sitios de muestreo de cada bosque deberían agruparse y diferenciarse de los otros. Se utilizó la prueba de correlación por rangos de Spearman para analizar si la asociación lineal entre el área basal total y el número de productos maderables era significativa.

\section{RESULTADOS Y DISCUSIÓN}

\section{Estructura poblacional de las unidades boscosas}

Bosque 1: esta unidad de vegetación presentó una densidad promedio de 181,4 ind ha-1 (117 adultos). El $35 \%$ de los individuos estuvieron comprendidos en las clases de regeneración, siendo esta la menor proporción de juveniles de las cuatro comunidades. La distribución de frecuencias diamétricas es más homogénea que en el resto de los bosques, existiendo algarrobos hasta los $110 \mathrm{~cm}$ de DAB (Fig. 3). La densidad de juveniles varió entre 10 y 143,3 ind $\mathrm{ha}^{-1}$, constituyendo la mayor variabilidad entre sitios de las cuatro comunidades estudiadas (coeficiente de variación, $\mathrm{CV}=$ 0,79) (Tabla 1). Esto indicaría que la renovación no es homogénea sino que podría estar dependiendo de micrositios adecuados 
para el establecimiento (Villagra \& Villalba 2001). La altura promedio de los árboles adultos fue de $5,7 \mathrm{~m}$, siendo el bosque con mayor densidad de árboles de más de $6 \mathrm{~m}$. El diámetro de copas promedio de los adultos fue de $5,3 \mathrm{~m}$ y la cobertura del $32,3 \%$. La mitad de los adultos poseía un solo fuste, forma erecta y se ubicó en la categoría de árboles sanos (Fig. 4).

Bosque 2: la densidad fue de 155 ind ha-1, variando entre 103,3 y 186,6. El porcentaje de juveniles fue del $37 \%$. La estructura diamétrica (de forma escalonada), mostró una marcada concentración de individuos entre los 2,5 y $17,5 \mathrm{~cm}$ de $\mathrm{DAB}$. No se observaron árboles de más de $45 \mathrm{~cm}$ de DAB (Fig. 3). La altura promedio de los adultos fue de $3,9 \mathrm{~m}$. El diámetro de copas $4 \mathrm{~m}$ y la cobertura del 20,3 $\%$. El $47 \%$ de los árboles adultos presentó un solo fuste y el $61 \%$ fueron erectos. El $93 \%$ de los fustes fueron menores a $1 \mathrm{~m}$ de alto. El 79 $\%$ de los individuos presentaron buen estado sanitario (Fig. 4).
Bosque 3: este bosque presentó la mayor densidad de árboles $\left(233,3\right.$ ind $\mathrm{ha}^{-1}$, variando entre 163,3 y 426,6 ind ha-1), con el $46 \%$ de adultos. Al igual que en el Bosque 1 , se destacó la alta variabilidad observada en la densidad de juveniles $(\mathrm{CV}=0,77)$. La altura promedio de los árboles adultos fue de $4,3 \mathrm{~m}$ y su diámetro de copa promedio de 4,5 m. Estos parámetros que caracterizan a los individuos indican baja variabilidad en su morfología $(\mathrm{CV}=0,12$ y 0,13 respectivamente $) . \mathrm{La}$ cobertura que alcanzó $P$. flexuosa en este bosque fue del 23,7 \%. Las mayores frecuencias diamétricas se dieron en las clases menores indicando una mejor regeneración con respecto a las otras unidades boscosas. Entre los adultos se observó un pico a los 20 cm de DAB (Fig. 3). Esta unidad presentó la menor proporción de individuos con buen estado sanitario (39\%) y $14 \%$ de árboles erectos, indicando una menor calidad forestal de la población (Fig. 4).

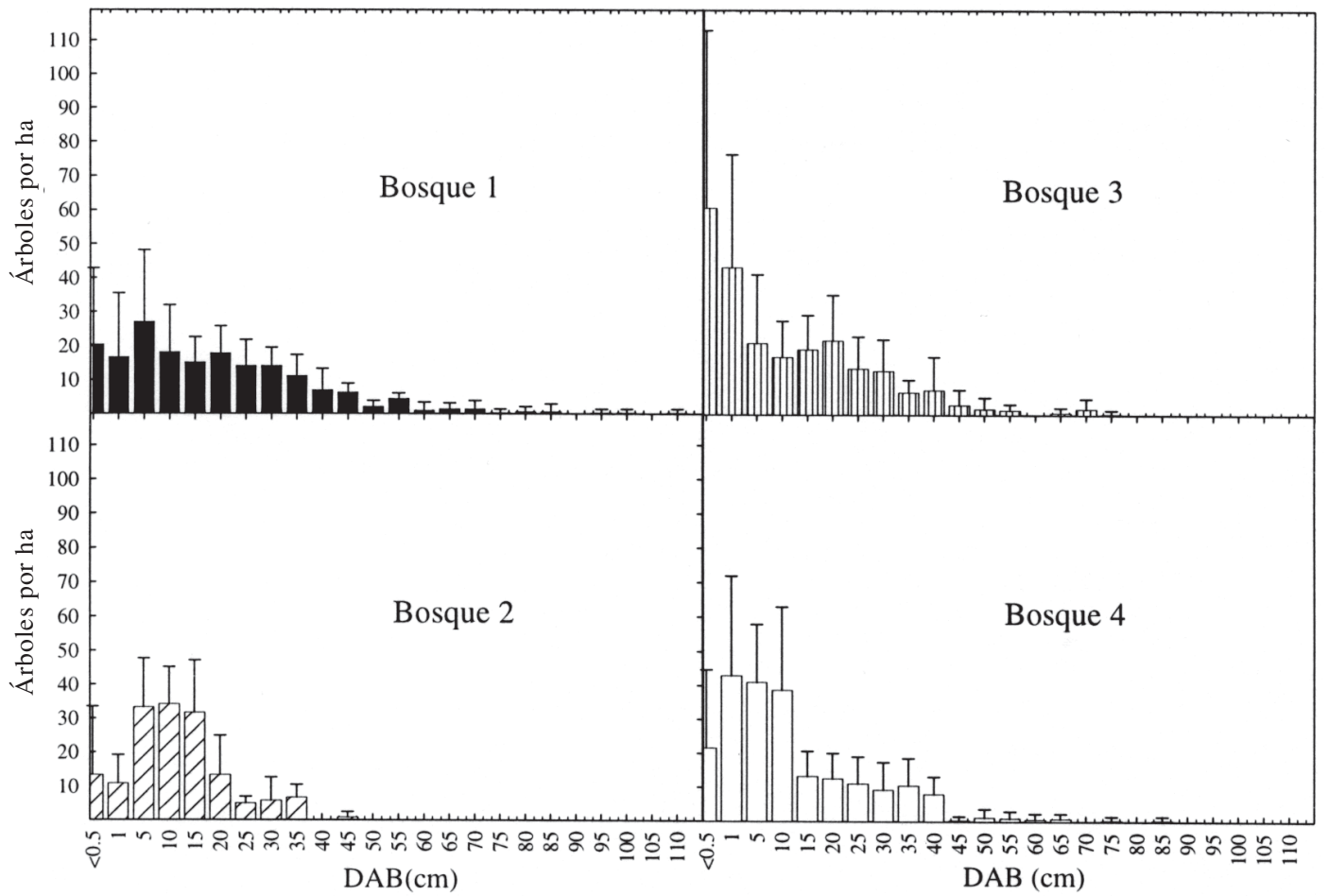

Fig. 3: Estructura diamétrica de los bosques 1, 2, 3 y 4. Las barras representan medias y las líneas errores estándar.

Diametric structure of forests 1, 2, 3 and 4. Bars represent means and lines standard errors. 
TABLA 1

Densidad total, de juveniles y árboles adultos (diámetro basal $>7,5 \mathrm{~cm}$ ), altura, diámetro de copa y cobertura de los algarrobos adultos en los cuatro bosques. Se presentan los datos de cada sitio como medias, desviaciones estándar (DE) y coeficientes de variación (CV)

Total density, of saplings and adult trees (basal diameter $>7.5 \mathrm{~cm}$ ), height, diameter of crown and coverage of the adult trees in the four forest. Data are given for every site as means, standard deviations (SD) and, coefficients of variation (CV)

\begin{tabular}{|c|c|c|c|c|c|}
\hline Variable & Parámetro & Bosque 1 & Bosque 2 & Bosque 3 & Bosque 4 \\
\hline \multirow[t]{3}{*}{ Densidad total (ind ha-1) } & Media & 181,4 & 155,0 & 233,3 & 214,8 \\
\hline & $\mathrm{DE}$ & 49,0 & 36,2 & 79,9 & 64,9 \\
\hline & $\mathrm{CV}$ & 0,27 & 0,23 & 0,34 & 0,30 \\
\hline \multirow[t]{3}{*}{ Densidad juveniles (ind ha ${ }^{-1}$ ) } & Media & 64,0 & 57,5 & 125,2 & 105,6 \\
\hline & $\mathrm{DE}$ & 50,4 & 16,6 & 96,9 & 56,9 \\
\hline & $\mathrm{CV}$ & 0,79 & 0,29 & 0,77 & 0,53 \\
\hline \multirow[t]{3}{*}{ Densidad adultos (ind $\mathrm{ha}^{-1}$ ) } & Media & 117,4 & 97,5 & 108,1 & 109,15 \\
\hline & $\mathrm{DE}$ & 26,8 & 29,1 & 41,7 & 37,1 \\
\hline & $\mathrm{CV}$ & 0,22 & 0,30 & 0,38 & 0,34 \\
\hline \multirow[t]{3}{*}{ Altura (m) } & Media & 5,7 & 3,9 & 4,5 & 4,1 \\
\hline & $\mathrm{DE}$ & 0,6 & 1,4 & 0,5 & 0,4 \\
\hline & $\mathrm{CV}$ & 0,12 & 0,31 & 0,12 & 0,21 \\
\hline \multirow[t]{3}{*}{ Diámetro de la copa (m) } & Media & 5,3 & 4,0 & 4,8 & 4,36 \\
\hline & $\mathrm{DE}$ & 0,8 & 0,3 & 0,6 & 0,9 \\
\hline & $\mathrm{CV}$ & 1,6 & 0,07 & 0,13 & 0,21 \\
\hline \multirow[t]{3}{*}{ Cobertura $(\%)$} & Media & 32,3 & 20,3 & 23,7 & 18,0 \\
\hline & $\mathrm{DE}$ & 11,9 & 13,9 & 7,2 & 5,9 \\
\hline & $\mathrm{CV}$ & 0,36 & 0,68 & 0,30 & 0,32 \\
\hline Número de sitios por bosque & & 9 & 4 & 9 & 8 \\
\hline Número de parcelas por bosque & & 27 & 12 & 27 & 21 \\
\hline
\end{tabular}

Bosque 4: la densidad media del mismo fue de 214,8 ind $\mathrm{ha}^{-1}$, variando la misma entre 126,6 y 323,3 algarrobos ha $^{-1}$. Los juveniles y adultos presentaron una densidad promedio similar, 49 y $51 \%$ respectivamente. Los árboles adultos tuvieron en este bosque una altura media de $4,1 \mathrm{~m}$, la cobertura promedio fue del $18 \%$. Las mayores frecuencias se dieron en las clases diamétricas de 2,5 a $12,5 \mathrm{~cm}$ de DAB (40 ind $\left.\mathrm{ha}^{-1}\right)$, encontrándose menos representadas las clases mayores a $12,5 \mathrm{~cm}$ (Fig. 3). Esto sugiere un bosque en recuperación con escasos individuos longevos. El $35 \%$ de los árboles adultos eran erectos y poseían un solo fuste, el $60 \%$ buen estado sanitario (Fig. 4). La cobertura de $P$. flexuosa en este bosque fue la menor de todas las unidades boscosas $(18 \%)$.

\section{Análisis de ordenación de los sitios de muestreo}

La ordenación de los sitios de muestreo en función de sus estructuras diamétricas mostró que la componente principal 1 se relaciona positivamente con la proporción de árboles de gran desarrollo, agrupando los sitios con mayor proporción de árboles de más de $25 \mathrm{~cm}$ de diámetro basal a la derecha del Eje 1 (valores positivos), mientras que a la izquierda se ubican los sitios con mayor proporción de juveniles (Fig. 5). Todos los sitios del Bosque 1 se ubicaron en los valores positivos del eje 1 , confirmando que esta unidad boscosa está caracterizada por la dominancia de árboles más grandes, con los mayores DAB, altura y cobertura, y menor regeneración. Los bosques 2 y 4 se ubicaron en los valores más negativos del 
eje 1 indicando que están caracterizados por la dominancia de individuos de clases diamétricas menores $(<12,5 \mathrm{~cm}$ de DAB). El Bosque 4, presentó árboles de menor altura y diámetro de copa. Por su parte, los sitios del Bosque 3 no se agrupan claramente sino que se distribuyen ampliamente a lo largo de los ejes uno y dos de la ordenación, mezclándose entre los sitios de las otras tres unidades boscosas. Esto indicaría una mayor variabilidad entre los sitios de este bosque desde el punto de vista poblacional. La dificultad de relacionar esta unidad de vegetación con la estructura diamétrica de la población de $P$. flexuosa, podría relacionarse con que la clasificación usada para diferenciar los distintos tipos de bosques se basó en los tipos referenciados en el mapa de vegetación que diferencia las unidades a partir de una síntesis de la reflectancia de toda la vegetación y no solo de su estrato arbóreo (González-Loyarte et al. 2000). De este modo en los bosques abiertos, la cobertura de especies arbustivas y herbáceas tiene una participación muy importante en la clase espectral identificada. Del análisis de la distribución de los sitios del Bosque 3 en la ordenación, surge que los sitios muestreados al norte se ubican cercanos al Bosque 1, mientras que los ubicados más al sur se ubican más cercanos al Bosque 4. Es posible que la variabilidad observada en el Bosque 3 sea producto de un gradiente de uso ya que el Bosque 4 es el que presentó más evidencia de uso forestal y el Bosque 1 el que menos evidencia de uso presentó.
Bosque 1

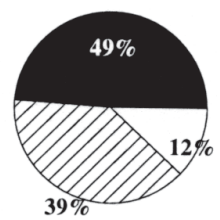

(A)

(B)

(C)
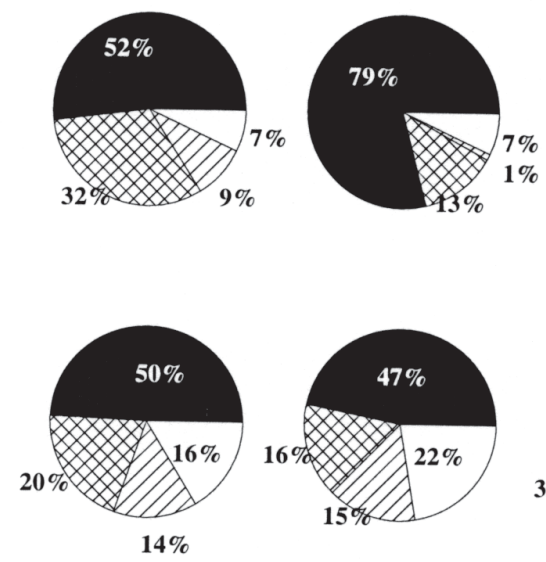

Bosque 4
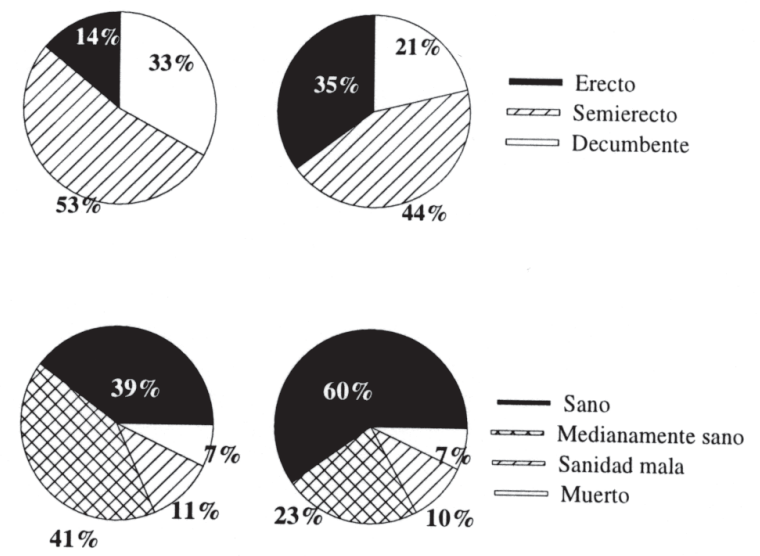

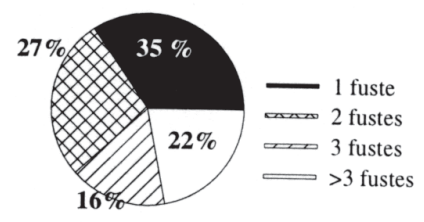

$14 \%$
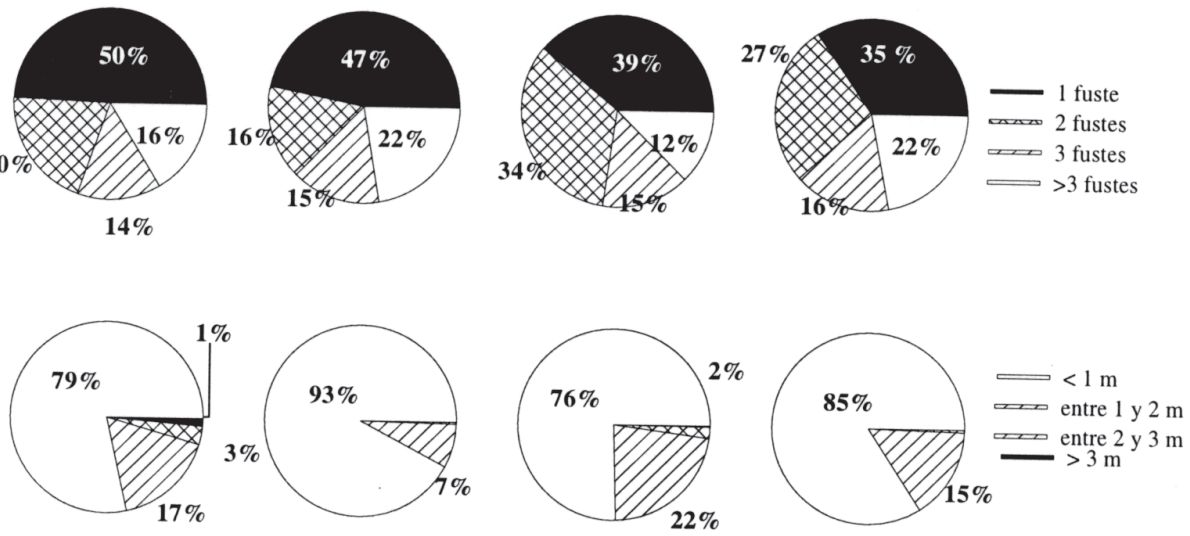

(D)

Fig. 4: Proporción de árboles según su forma (A); estado sanitario (B); número de fustes (C) y altura de los fustes en los cuatro bosques estudiados (D).

Proportion of the trees according to: shape (A); sanitary condition (B); number of stems (D), and height of the stems (D). 


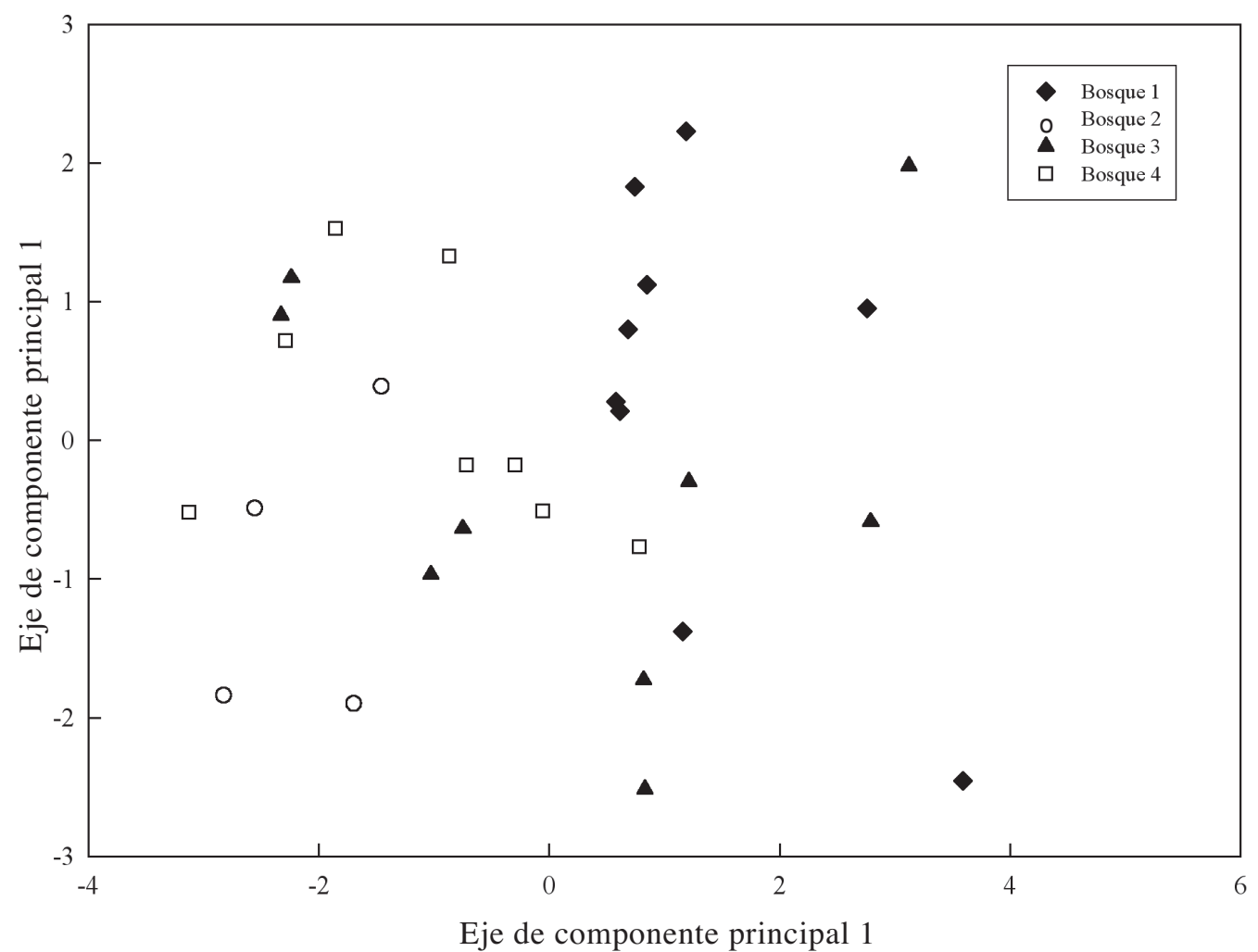

Fig. 5: Análisis de componentes principales según el DAB para todos los sitios de muestreo. Se relevaron 30 sitios: nueve en el Bosque 1, cuatro en el Bosque 2, nueve en el Bosque 3 y ocho en el Bosque 4.

Analysis of principal components according to the DAB for all the sampling sites. Thirty sites were examined: nine in Woodland 1, four in Woodland 2, nine in Woodland 3 and eight in Woodland 4.

\section{Productos maderables}

En los cuatro bosques analizados, los postes y medio postes fueron los productos forestales más encontrados, mientras que las vigas presentaron un muy bajo número. Los bosques 1 y 3 son los que presentaron mayor disponibilidad de productos forestales; en total sumando todo los tipos de postes se encontraron 36,3 postes por ha en el Bosque 3 y 32,5 en el Bosque 1. Por el contrario, los productos forestales existentes en el Bosque 2 y 4 son sumamente escasos. Este último presenta una historia de uso en la que se extrajeron postes y leña gracias a la buena accesibilidad hasta principios del siglo XX (Roig et al. 1992). La madera para aserrío representa solo una proporción muy baja del total, (4,8 vigas por ha en el Bosque 1). Al relacionar el área basal total con el número de productos forestales por ha, se observa que cuanto mayor es el área basal total, es mayor la cantidad de productos forestales. Los sitios del Bosque $1 \mathrm{se}$ agrupan hacia los valores más positivos del área basal y del número de productos forestales por ha, intercambiándose con algunos sitios del Bosque 3. Mientras que los bosques 2 y 4 se agrupan en zonas con baja área basal y menos de 10 productos forestales por ha (Fig. 6). La asociación lineal entre variables fue significativa (correlación por rangos de Spearman, $\left.r_{s}=0,67 ; n=30 ; P=0,0001\right)$.

No se disponen para el área de ecuaciones alométricas que permitan estimar el volumen de madera, aunque en otra área del Monte (Pipanaco, Catamarca) este se relaciona en forma lineal con el área basal de la parcela (Cony \& Villagra resultados no publicados). Además se ha observado que la proporción de madera destinada a leña varía entre el $100 \%$ en 
unidades en recuperación hasta el $60 \%$ en los sitios con árboles de porte erecto y de mayor DAB. Teniendo en cuenta el bajo número de productos forestales por ha en relación a la densidad del bosque que se observaron en este trabajo, se postula que la proporción de madera potencialmente útil para un producto forestal de mayor valor comercial que la leña es muy baja.

Se puede observar en la Fig. 6 un sitio con $21 \mathrm{~m}^{2}$ de área basal y 40 productos ha-1 que está fuera del rango de los otros valores. Este sitio de muestreo posee los árboles más grandes (> $85 \mathrm{~cm}$ de DAB), sin embargo, el crecimiento de estos árboles es decumbente y la sanidad es menor, lo que reduce la posibilidad de encontrar postes en los mismos, esto sugeriría que el envejecimiento del bosque reduce la calidad de los productos forestales.
Análisis comparativo y consideraciones para el manejo

La descripción de la estructura y estado de conservación, permiten conocer la situación actual de los bosques en estudio e integran la base de cualquier proyecto de manejo. Combinando el estado sanitario y la forma de los individuos se puede determinar qué usos puede atribuirse a la madera presente en los árboles estudiados. La madera de los individuos sanos, con fuste recto y la altura adecuada, puede destinarse a la fabricación de muebles o producción de postes. Si los árboles son de forma semierecta, con buen estado sanitario, parte de la madera puede ir a aserrío y el resto a leña y carbón (Calzon-Adorno \& Ortin-Vujovich 2000).

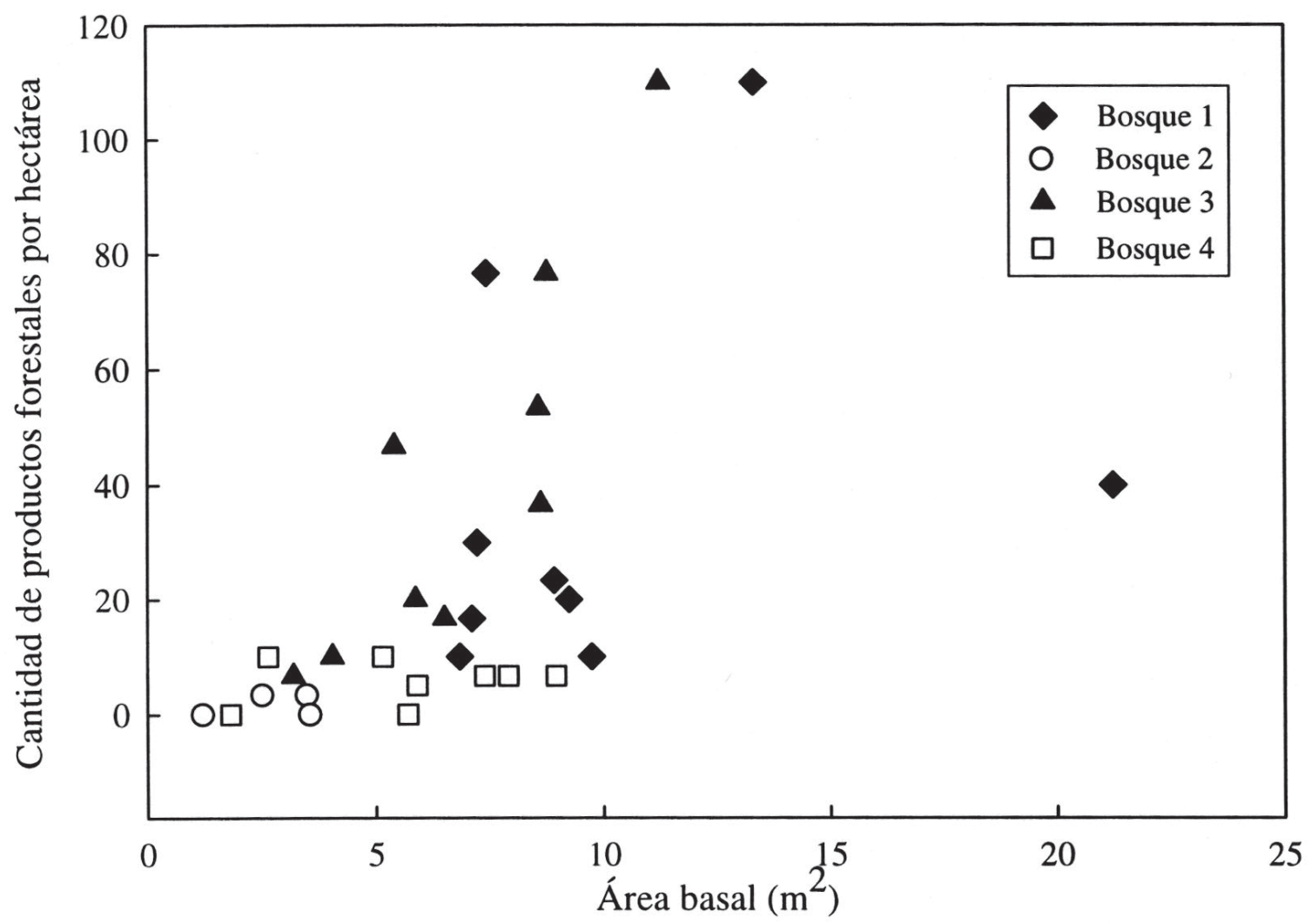

Fig. 6: Relación entre la cantidad de productos forestales y el área basal de los sitios de muestreo. La cantidad de leña presente en cada sitio no fue cuantificada en este estudio.

Relationship between the quantity of wood products and the basal area of the sample sites. The quantity of fuelwood in every site was not quantified in this study. 
Un aspecto a tener en cuenta es el desarrollo de los árboles. En las cuatro unidades estudiadas, la altura media de los algarrobos se encuentra entre los 3,9 y $5,7 \mathrm{~m}$; teniendo el Bosque 1 la mayor proporción de individuos de más de $6 \mathrm{~m}$. Comparando con otros bosques del Monte, en Pipanaco ${ }^{1}$ (Catamarca) la altura promedio de los bosques de $P$. flexuosa alcanza los $9 \mathrm{~m}$ y en Ñacuñán los 3,7 m (Villagra \& Villalba 2001). Estas diferencias de alturas medias entre los bosques del norte (Pipanaco) y los de la zona central del Monte (Telteca y Ñacuñán) podrían deberse a consecuencias adaptativas de esta especie con respecto a las bajas temperaturas soportadas por las plantas (mayor proporción de días con heladas en el centro del Monte), ya que se ha observado en otras especies de Prosopis que la presencia de múltiples fustes y baja altura se debe a la destrucción del meristema apical por el accionar de animales, procesos físicos o heladas (Fisher 1977).

Por otra parte, mientras que en Pipanaco los árboles de un solo fuste representan al $83 \%$ de la población y la altura de los fustes (entre 2 y $5 \mathrm{~m}$ ) permitiría un aprovechamiento sustentable de productos madereros de alto valor económico, en el noreste de Mendoza el bosque con mayor proporción de individuos de un solo fuste es el Bosque 1 (50 \%), a lo que se le agrega que la mayoría de los fustes se ramifican antes del metro. Desde el punto de vista forestal esto representa un problema que disminuye la calidad de los productos y la rentabilidad por lo que debería ponerse a prueba si trabajos silviculturales, como las podas de formación, pueden mejorar este atributo estructural del bosque.

La estructura poblacional de los bosques y la forma de los árboles sugieren que no es posible la planificación de un manejo tendiente a la extracción de productos forestales de alto valor, sino que la disponibilidad se limita a leña o carbón, y una baja producción de postes exclusivamente en los bosques 1 y 3 . El desarrollo de ecuaciones alométricas que

\footnotetext{
${ }^{1}$ CONY MA, PE VILlagra \& JA ALVAREZ (2004) Producción actual y potencial de leña y madera en algarrobales del Monte, Argentina. Pautas de manejo y aprovechamiento sustentables. Segunda Reunión Binacional de Ecología, Mendoza, Argentina.
}

permitan la cuantificación de la biomasa forestal total para cada unidad boscosa y la estimación de la existencia actual de leña seca completaría una descripción detallada del estado actual del bosque. Sin embargo, para poder determinar el potencial de uso forestal de la zona es necesario calcular la productividad maderera anual de la población y los turnos de cortabilidad. El uso de técnicas dendrocronológicas permite obtener la productividad anual del bosque a través de las mediciones del ancho de los anillos de crecimiento anual de los árboles, las que se pueden expresar como crecimiento radial y/o basal y ser transformadas luego a incremento en peso o volumen aplicando ecuaciones alométricas (Assman 1970). Estudios previos demuestran la presencia de anillos de crecimiento anuales en esta especie y sugieren que es posible el uso de aproximaciones dendroecológicas en la planificación del manejo de estos bosques (Villalba \& Boninsegna 1989, Villalba et al. en prensa). En otros bosques del Monte se ha observado que el crecimiento corriente presenta una gran variabilidad intrapoblacional e interpoblacional (Villagra et al. en prensa, Villalba et al. en prensa). Además, estas técnicas han permitido observar una fuerte relación entre cronologías de ancho de anillos de crecimiento y variables climáticas, en otras especies de Prosopis, como $P$. ferox en los desiertos subtropicales de alta montaña del noroeste argentino (Morales et al. 2001). Como complemento de los datos obtenidos, sería muy importante contar con la estructura de edades del bosque, para determinar cuál es la regeneración y qué relación guarda con el clima y la historia de uso del área de estudio.

Los datos obtenidos en este estudio, la importancia de $P$. flexuosa como especie estructuradora del ecosistema (Rossi \& Villagra 2003) y la forma en que los pobladores se relacionan con el bosque, sugieren analizar la posibilidad del manejo silvopastoril, siendo necesario evaluar la productividad de los estratos arbóreo (follaje y frutos), arbustivo y herbáceo. En la zona existen trabajos que indican que la presencia del P. flexuosa aumenta la receptividad ganadera por un aumento en la cobertura de especies palatables bajo su dosel (Cesca 2003). Por último, deberían revisarse los aspectos legales vigentes, basándose en 
fundamentos técnicos de productividad del bosque y su capacidad de regeneración. Una posibilidad a tener en cuenta es la utilización de la leña seca y leña campana (nombre que se le da en la zona a las ramas interiores que mueren por el sombreado de las ramas superiores) (Roig 1985). La proporción de árboles muertos o con mala sanidad es baja, aunque deberían analizarse las ventajas y consecuencias de realizar una corta de saneamiento y el aprovechamiento de los mismos para leña.

\section{AGRADECIMIENTOS}

Los autores desean expresar su agradecimiento a Ricardo Villalba por sus valiosas sugerencias y a Gualberto Zalazar, Belén Lana, Germán Berra y Silvia Delgado por su colaboración en las tareas de campo. Este trabajo ha sido financiado por la Agencia Nacional de Promoción Científica y Tecnológica de Argentina (PICT 03220) y por el Interamerican Institute for Global Change Research (IAI).

\section{LITERATURA CITADA}

ABRAHAM EM (2000) Geomorfología de la Provincia de Mendoza. En: Abraham EM \& F Rodríguez Martínez (eds) Recurso y problemas ambientales de la zona árida. Provincias de Mendoza, San Juan y La Rioja: 29-48. Junta de Gobierno de Andalucía Universidades y Centros de Investigación de la Región Andina Argentina, Mendoza. Argentina.

ABRAHAM EM \& MR PRIETO (1999) Vitivinicultura y desertificación en Mendoza. En: García Martínez B (ed) Estudios de historia y ambiente en América: Argentina, Bolivia, México, Paraguay: 109-135. Instituto Panamericano de Geografía e Historia (IPGH) - Colegio de México, México.

ASSMAN E (1970) The principles of forest yield study. Pergamon Press, New York, New York, USA. 506 pp.

BAGNOULS F \& H GAUSSEN (1957) Climats biologiques et leur classification. Annales de Géographie (Francia) 355: 193-220.

BRAUN-BLANQUET J (1979) Fitosociología. Blume Ediciones, Madrid, España. 819 pp.

CALZON-ADORNO ME \& AE ORTIN-VUJOVICH (2000) Análisis de la estructura poblacional de un bosque de algarrobo (Prosopis spp.) en Cafayate, Salta, Argentina. En: Jiménez P, C Talavera Delgado, L Villegas Paredes, A Ortega Paredes \& F Villasante Benavides (eds) Memorias del IV Congreso Latinoamericano de Ecología: ecología y desarrollo sostenible: reto de América Latina para el tercer milenio: 145-148. Instituto Regional de Ciencias Ambientales y UNESCO, Arequipa, Perú.

CESCA E (2003) Efecto de Prosopis flexuosa sobre las especies palatables y su importancia para el manejo pastoril de los algarrobales del noreste de Mendoza. Tesis de Licenciatura, Universidad del Congreso, Mendoza, Argentina. $61 \mathrm{pp}$.

ESTRELLA HA, VA HERAS \& VA GUZZETA (1979) Registro de elementos climáticos en áreas críticas de la provincia de Mendoza. Instituto Argentino de Investigaciones en Zonas Áridas (Argentina), Cuaderno Técnico 1-79: 49-71.

FAO (1999) Parte I: situación y perspectivas de la conservación y desarrollo de los bosques. Situación de los bosques del mundo 1999: FAO, Roma, Italia. $47 \mathrm{pp}$.

FISHER CE (1977) Mesquite and modern man in southwestern North America. En: Simpson BB (ed) Mesquite, its biology in two desert shrub ecosystems: 177-188. Downden, Hutchinson and Ross Inc., Stroudsburg, Pennsylvania, USA.

GOBIERNO DE MENDOZA (1997a) Ley Provincial 2088. Creación de la Administración Provincial de Bosques. Adhesión de la Ley Nacional 13273. Dirección de Recursos Naturales Renovables \& Ministerio de Ambiente y Obras Públicas, Compendio de Leyes y Reglamentaciones. Comunicación Social del Ministerio de Ambiente y Obras Públicas Mendoza. Mendoza, Argentina. 175 pp.

GOBIERNO DE MENDOZA (1997b) Resolución No 854. Dirección de Bosques y Parques Provinciales. Dirección de Recursos Naturales Renovables \& Ministerio de Ambiente y Obras Públicas, Compendio de Leyes y Reglamentaciones. Comunicación Social del Ministerio de Ambiente y Obras Públicas, Mendoza, Argentina. 175 pp.

GOBIERNO DE MENDOZA (1997c) Resolución No 1609. Dirección de Recursos Naturales Renovables. Dirección de Recursos Naturales Renovables \& Ministerio de Ambiente y Obras Públicas, Compendio de Leyes y Reglamentaciones. Comunicación Social del Ministerio de Ambiente y Obras Públicas, Mendoza, Argentina. 175 pp.

GOBIERNO DE MENDOZA (1997d) Ley Provincial 4609. Protección de la Flora de la Provincia. Dirección de Recursos Naturales Renovables \& Ministerio de Ambiente y Obras Públicas, Compendio de Leyes y Reglamentaciones. Comunicación Social del Ministerio de Ambiente y Obras Públicas, Mendoza, Argentina. 175 pp.

GONZÁLEZ-LOYARTE MM (1992) Reserva faunística y florística Telteca. Las Áreas Protegidas de la Provincia de Mendoza: Instituto Argentino de Investigaciones en Zonas Áridas (IADIZA), Mendoza, Argentina. $175 \mathrm{pp}$

GONZÁLEZ-LOYARTE MM, M MENENTI, P WEIDEMA, FA ROIG \& M BARTON (2000) Mapping vegetation degradation applying remotely sensed data in the arid zones of Argentina. The northeastern plain of Mendoza. Proceedings of United Nations/International Astronautical Federation Workshop on "Operational Strategy for Sustainable Development using Space". United Nations Committee on the Peaceful Uses of Outer Space and Office for Outer Space Affairs, Sao José dos Campos, Brazil. 175 pp.

MORALES MS, R VILLALBA, HR GRAU, PE VILLAGRA, JA BONINSEGNA, A RIPALTA \& L PAOLINI (2001) Potencialidad de Prosopis ferox Griseb (Leguminosae, subfamilia: Mimosoideae) para estudios dendrocronológicos en desiertos subtropicales de alta montaña. Revista Chilena de Historia Natural 74: 889-896. 
MORELLO J (1958) La Provincia Fitogeográfica del Monte. Opera Lilloana (Argentina) 2: 5-115.

PASIECZNIK NM, P FELKER, PJC HARRIS, LN HARSH, G CRUZ, JC TEWARI, K CADORET \& LJ MALDONADO (2001) The Prosopis julifloraProsopis pallida complex: a Monograph. Henry Doubleday Research Association, Coventry, United Kingdom. 162 pp.

ROIG FA (1985) Árboles y bosques de la región árida centro oeste de la Argentina (Provincias de Mendoza y San Juan) y sus posibilidades silvícolas. Forestación en zonas áridas y semiáridas. Segundo encuentro regional del Centro Internacional de Investigaciones para el Desarrollo (CIID), América Latina y el Caribe. Instituto Forestal de Chile (INFOR), Santiago, Chile.

ROIG FA (1993) Informe nacional para la selección de germoplasma en especies del género Prosopis de la República Argentina. Contribuciones mendocinas a la Quinta Reunión de Regional para América Latina y el Caribe de la Red de Forestación del Centro Internacional de Investigaciones para el Desarrollo (CIID). Conservación y Mejoramiento de Especies del Género Prosopis. Instituto Argentino de Investigaciones de las Zonas Áridas (IADIZA)Centro Regional de Investigaciones Científicas y Tecnológicas (CRICYT)- Centro Internacional de Investigaciones para el Desarrollo (CIID). Mendoza, Argentina. 153 pp.

ROIG FA, A BERRA, M GONZÁLEZ LOYARTE, E MARTÍNEZ CARRETERO \& C WUILLOUD (1992) La Travesía de Guanacache, tierra forestal. Multequina (Argentina). 1: 83-91.

ROSSI BE \& PE VILLAGRA (2003) Effects of Prosopis flexuosa on soil properties and the spatial pattern of understory species in arid Argentina. Journal of Vegetation Science 14: 543-550

VILLAGRA PE \& R VILLALBA (2001) Estructura poblacional del algarrobal de la Reserva de Ñacuñán. En: Claver S \& S Roig-Juñent (eds) El desierto del Monte: la Reserva de la Biósfera de Ñacuñán: 71-75. Instituto Argentino de Investigaciones de las Zonas Áridas (IADIZA)Man and the Biosphere Programme (MAB)- United Nations Educational Scientific and Cultural Organisation (UNESCO). Mendoza, Argentina.

VILLAGRA PE, JA BONINSEGNA, JA ÁLVAREZ, MA CONY, E CESCA \& R VILLALBA (en prensa) Dendroecology of Prosopis flexuosa woodlands in the Monte desert: implications for their management. Dendrochronologia.

VILLAGRA PE, MA CONY, NG MANTOVÁN, BE ROSSI, MM GONZÁLEZ LOYARTE, R VILLALBA \& L MARONE (2004) Ecología y manejo de los algarrobales de la Provincia Fitogeográfica del Monte. En: Arturi MF, JL Frangi \& JF Goya (eds) Ecología y manejo de bosques nativos de Argentina. Presentación multimedia en CD, Editorial Universidad Nacional de La Plata, La Plata, Argentina.

VILLALBA R \& JA BONINSEGNA (1989) Dendrochronological studies on Prosopis flexuosa D.C. International Association of Wood Anatomists (IAWA), Bulletin (Holanda) 10: 155-160.

VILLALBA R, PE VILLAGRA, JA BONINSEGNA, MS MORALES \& V MOYANO (en prensa) Dendroecología y dendroclimatología con especies del género Prosopis. Multequina (Argentina)

Editor Asociado: Julio Gutiérrez.

Recibido el 22 de abril de 2005; aceptado el 19 de agosto de 2005 